\title{
Reviving and Extending the Neutron Imaging Capabilities at the Penn State Breazeale Reactor
}

\author{
Robert Zboray ${ }^{1, \mathrm{a}^{*}}$ \\ ${ }^{1}$ Department of Mechanical and Nuclear Engineering, The Pennsylvania State University, 233 \\ Reber Building, University Park, PA 16802, USA \\ arzz65@psu.edu
}

Keywords: Neutron Imaging, TRIGA, Core Moderator, Bright Flash Imaging, MultiSpectral Imaging, Fast Neutron Imaging

\begin{abstract}
The Penn State Breazeale Reactor (PSBR), a 1 MW TRIGA type reactor has been utilized successfully for neutron imaging in the past. Presently a single beam line with a thermal spectrum is utilized for imaging, however the reactor is just undergoing a major refurbishment involving the installation of a new core moderator assembly. This enables the establishment of several new neutron beam lines around the reactor including three with a cold spectrum. We report here on the ongoing developments and future plans to revive and extend neutron imaging capabilities at PSBR.
\end{abstract}

\section{Introduction}

The Breazeale reactor operated by the Radiation Science and Engineering Center (RSEC) of the Pennsylvania State University, is a 1-MW TRIGA type reactor. It has been successfully utilized in the past for neutron imaging and its imaging beam line, which has been operational until very recently (mid 2018), has been upgraded on few occasions. The layout of this old imaging beam line is shown in Fig. 1. It was an ASTM E 544 Category 1 facility with a tangential collimator. It featured a steady neutron flux of $1.7^{*} 10^{7} \mathrm{n} / \mathrm{cm}^{2} / \mathrm{s}$ at full power and has an L/D collimation ratio of around 150 at the sample position. Further details on the beam line and on past imaging activities, can be found in [1] and [2].

A drawback of the old moderator and beam port design was that the beam ports, except for the imaging beam line \#4 on Fig. 1, were not axially aligned with the core center therefore could not feature the highest possible flux. This was related to a change of the fuel type of PSBR from high-enriched uranium to low-enriched TRIGA fuel. Furthermore, the imaging beam line \#4 had a pretty high gamma contribution. This was mainly attributed to the thermal neutron capture reaction by hydrogen in pool water which mainly takes place at the sides of the old coremoderator assembly (see Fig. 1) as pointed out in [3]. The high gamma background was not only disturbing for certain imaging applications (see below) but also for some other neutron irradiation techniques sporadically performed at the beam line.

Due to these deficiencies and in a broader context to enhance neutron science capabilities around the reactor, a conceptual design of a new core moderator assembly and new set of beam ports have been prepared using extensive Monte Carlo and thermalhydraulic simulations [3]. This has resulted in a crescent-shaped core-moderator assembly filled with D2O and a number of radial beam ports (see Fig. 2 left). The main advantages of this new design are: larger number and variety of beamlines aligned axially with the core, higher fluxes than in the existing beam line(s), improved $\mathrm{n} / \gamma$ ratios due to the reduced ${ }^{1} \mathrm{H}(\mathrm{n}, \gamma) \mathrm{H}^{2}$ reaction by the new moderator geometry and improved Cd ratios. The new thermal beam line NBP4 will be exclusively dedicated to neutron imaging. Some of the design parameters of this beam line are as follows: 
the thermal neutron flux is around $7 * 10^{7} \mathrm{n} / \mathrm{cm}^{2} / \mathrm{s}$ and the fast flux $(>2 \mathrm{MeV})$ is about $6 * 10^{6}$ $\mathrm{n} / \mathrm{cm}^{2} / \mathrm{s}$. These are unfiltered flux estimates. Depending on the type and thickness of the applied in-beam filters (Bi, sapphire) these values can decrease up to a factor five for thermal and a factor twenty for fast flux. Regarding flexible beam filtering and spectral shaping see the text below. The expected L/D collimation ratio is around 130 at the sample position ( $1 \mathrm{~m}$ away from the outer wall of the biological shielding). One of the new cold beam lines will also be partly used for neutron imaging. Furthermore, the exploratory free beam NBP2 could also be occasionally used for imaging related studies.
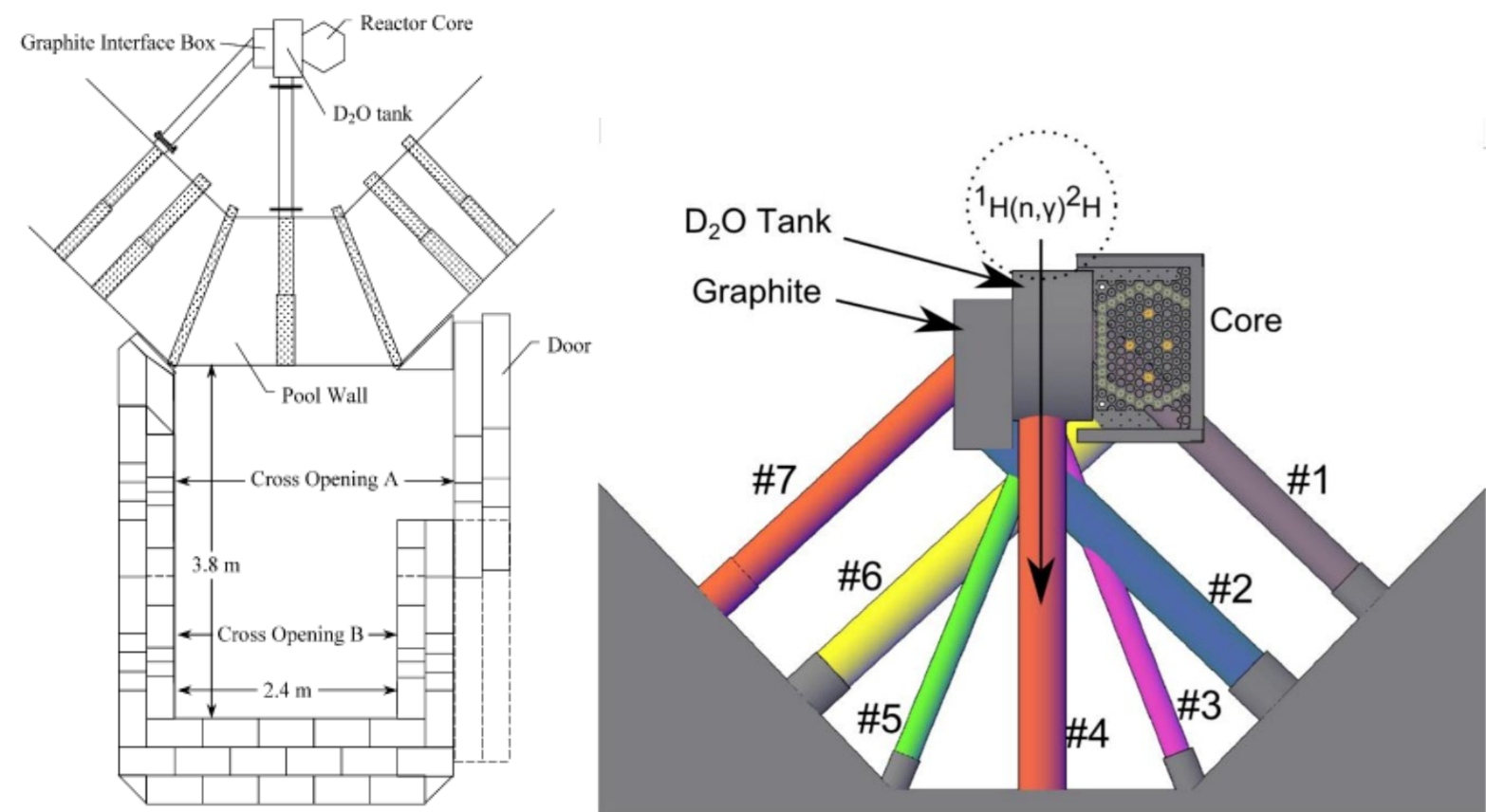

Fig. 1: Layout of the old neutron imaging beam line at the PSBR. Left: the beam cave is shown together with the beam port and the biological shield. Right: the old D2O moderator tank and the old beam ports. \#4 is the imaging beam line.
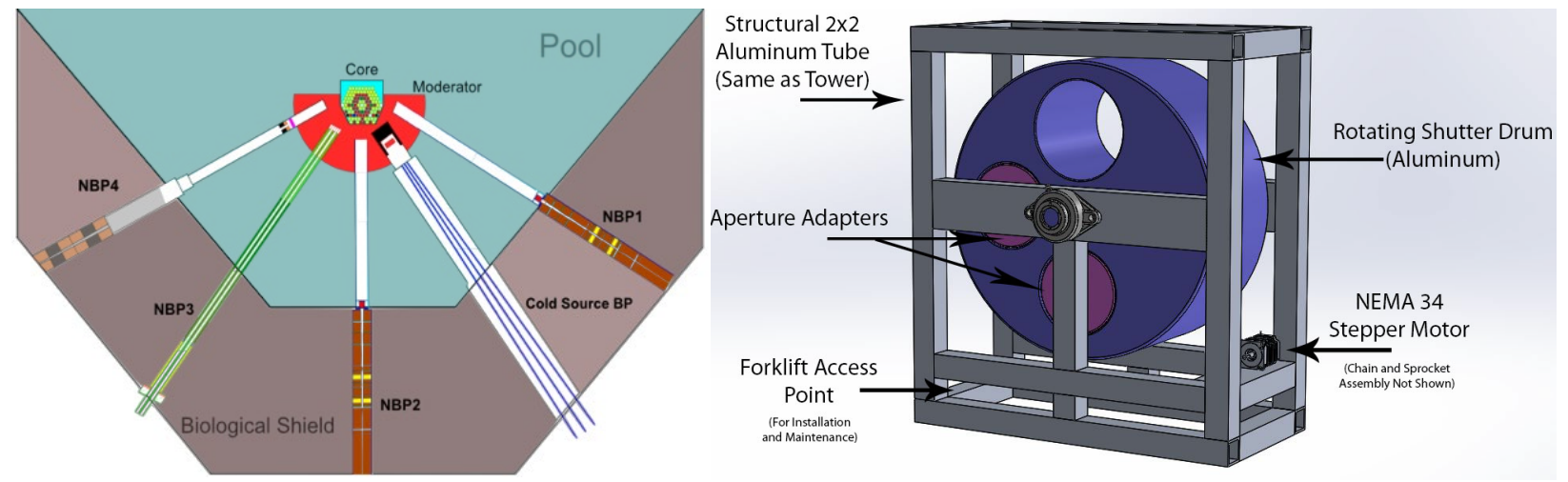

Fig. 2: Left: layout of the new core moderator assembly with the planned new radial beam port and beam lines. The image is taken from [3]. Right: Rotary beam shutter with several apertures enabling easy and fast shutting down of theNBP4 beam and also a flexible in-beam filtering. 


\section{Imaging technique developments at PSBR}

General thermal neutron imaging. Not long before the reactor refurbishment, we have started extensive developments to enhance the imaging capabilities and activities at PSBR. First, efficient state-of-the-art digital, camera-based detectors have been introduced. One such detector for general thermal neutron imaging purposes have been obtained from NeutronOptics [4] featuring a 200x250 $\mathrm{mm}^{2}$ field-of-view (FOV) and a CCD camera with a 1 inch Sony ICX694ALG EXview HAD CCD II chip with 2750x2200 pixels. Dark current is strongly reduced $\left(0.002 \mathrm{e} / \mathrm{pix} / \mathrm{s} @-10{ }^{\circ} \mathrm{C}\right)$ by thermo-electric cooling at $-35 \mathrm{C}$. The camera has 16 bit digital output and a high, $\sim 75 \%$ quantum efficiency (QE). The camera is coupled with a highresolution f/1.4 Fujinon C-mount lens. A photo of the camera box and the CCD is shown in Fig. 3.

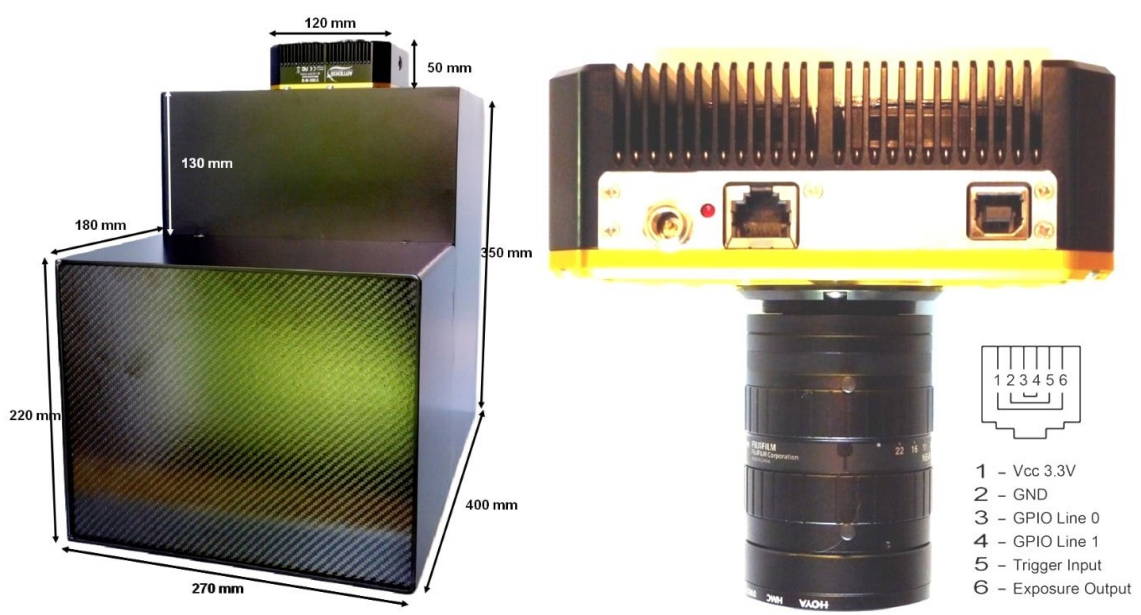

Fig. 3: Photo of the detector box showing its front (sensitive) face (left) and of the CCD camera (right).

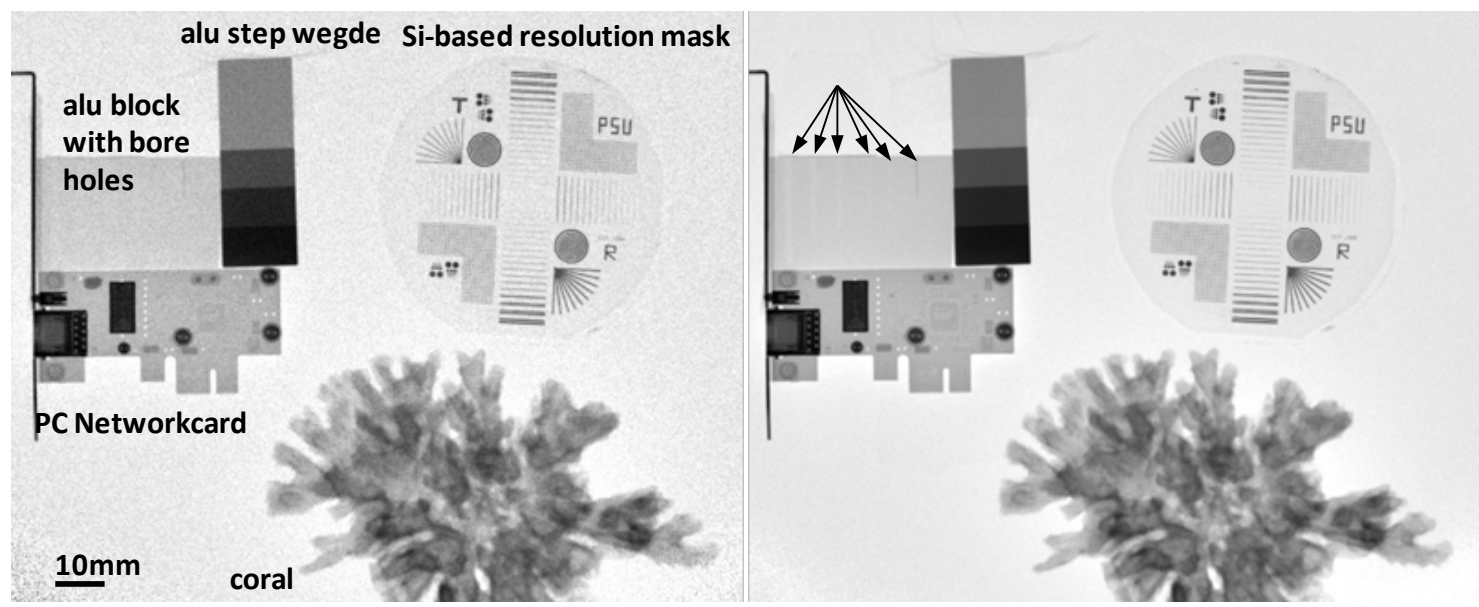

Fig. 4: Two images of several test objects obtained at the old thermal imaging beam line using the detector shown in Fig. 3 in conjunction with a high efficiency 400 um LiF/ZnS screen at only $10 \%$ power of the reactor with as short as 5 s exposure (left) and a longer 60s exposure (right).

Some example neutron images of several test objects are shown in Fig. 4 taken by the above detector in combination with a $400 \mu \mathrm{m}$ thick, high efficiency $\mathrm{LiF} / \mathrm{ZnS}(\mathrm{Cu})$ screen from Scintacor [5] on the detector box to illustrate imaging quality at the beamline using the above detector. The 
high detection efficiency of the screen enabled to take reasonable quality radiographs at only $10 \%$ reactor power and with exposure as low as 5s (see Fig. 4 left). The image shows visually observable noise but the overall quality is still reasonable (SNR=22.2) only the fine bore holes in the aluminum block go undetected due to the noise as is illustrated compared to Fig. 4 on the right, which is obtained for 60s exposure and with very good quality (SNR=62.1). The arrows indicated the bore holes that are from left to right 3.5, 2, 1.5, $1 \mathrm{~mm}$ in diameter in a $20 \mathrm{~mm}$ thick aluminum block and the rightmost, $1.5 \mathrm{~mm}$, is filled with a steel bar. The test objects in Fig. 4 represent different levels of structural complexity (from a simple aluminum step wedge to a PCB board of a PC network card and a highly structured coral) and cover a broad variety of material compositions (organics, metals, minerals etc.). Note that due to the thick screen the spatial resolution, is estimated based on the edge spread function of the upper part of the aluminum step wedge, is only around $500 \mathrm{um}$, however the primary purpose of the screen was to use it for bright flash imaging (see below).

Bright flash neutron imaging. TRIGA reactors, due to their special fuel composition, allow operation in pulse mode [9]. In pulse operation one of the control rods, in case of the Penn State reactor the transient rod, is rapidly ejected to a certain extent from the core with the help of a pneumatic system making the reactor prompt supercritical and engaging it on a power excursion. The power excursion is then mitigated by the strong prompt negative feedback from fuel temperature on reactivity brought about by the fuel composition (uranium mixed intimately with the zirconium hydride moderator) and the reactor power settles down to practically zero again. Peak power values can reach almost $1 \mathrm{GW}$ [6]. For the definition of reactivity in $\$$ and for details on prompt supercritical reactor physics the reader is referred to [9].

This paves the way towards high-speed neutron radiography for fast transient processes. Such processes require high acquisition rate potentially in the kilo frame per second (kfps) range to capture them with minimal motion blur. However, due the short exposure times a high neutron flux is needed to obtain images with acceptable signal-to-noise ratio and statistics.

We have recently demonstrated up to $4 \mathrm{kfps}$ bright flash radiography of a two-phase bubbly flow in a simple bubbler made of aluminum using the old imaging beam line. Some illustrative results are shown in Fig. 5. For this purpose, we have replaced the CCD camera with a legacy high-speed CMOS camera with special, high brightness optics. We report on the details of our bright flash imaging developments elsewhere.

Fast neutron imaging, multi-spectral imaging. Besides thermal neutron imaging we plan to develop fast neutron or multi-spectral imaging methods. This is will be implemented by designing the new thermal imaging beam line in the most flexible way. It will feature (re)moveable in-beam filters to enable multi spectrum imaging ranging from the standard thermal to epithermal and fast energy ranges. Typically, sapphire filters are applied in thermal beam lines to reduce both the gamma and mainly the fast neutron contribution. We plan to have a removable sapphire filter seated deep in the beam port not so far away from the new moderator tank.

We have recently tried to perform fast neutron imaging using the old beam line. The test object was a massive Li-fueled power source shown in Fig.6. The detector was equipped with a $3.8 \mathrm{~mm}$ thick PP/ZnS:Cu fast neutron imaging screen from RC Tritec AG, Switzerland [7]. The thermal content of the beam has been removed by applying a 1-cm thick Mirrobor (a borated rubber matt [8]) in-beam filter to avoid unnecessary activation of the sample and the detector. Even exposure times as high as 12 minutes delivered suboptimal image quality (see Fig.6), though the upper oxidized area can be clearly distinguished from the lower elemental Li parts. The low image quality was likely due to the insufficient fast flux in the old tangential beam line, that was looking into the middle of the $\mathrm{D}_{2} \mathrm{O}$ tank. Furthermore, a permanently placed sapphire 
filter was also present in the beam path of that beam line. For these reasons we plan the new beam line with a removable sapphire filter and the MCNP simulations also show that the new radial beam line will have a significantly higher native fast neutron content.

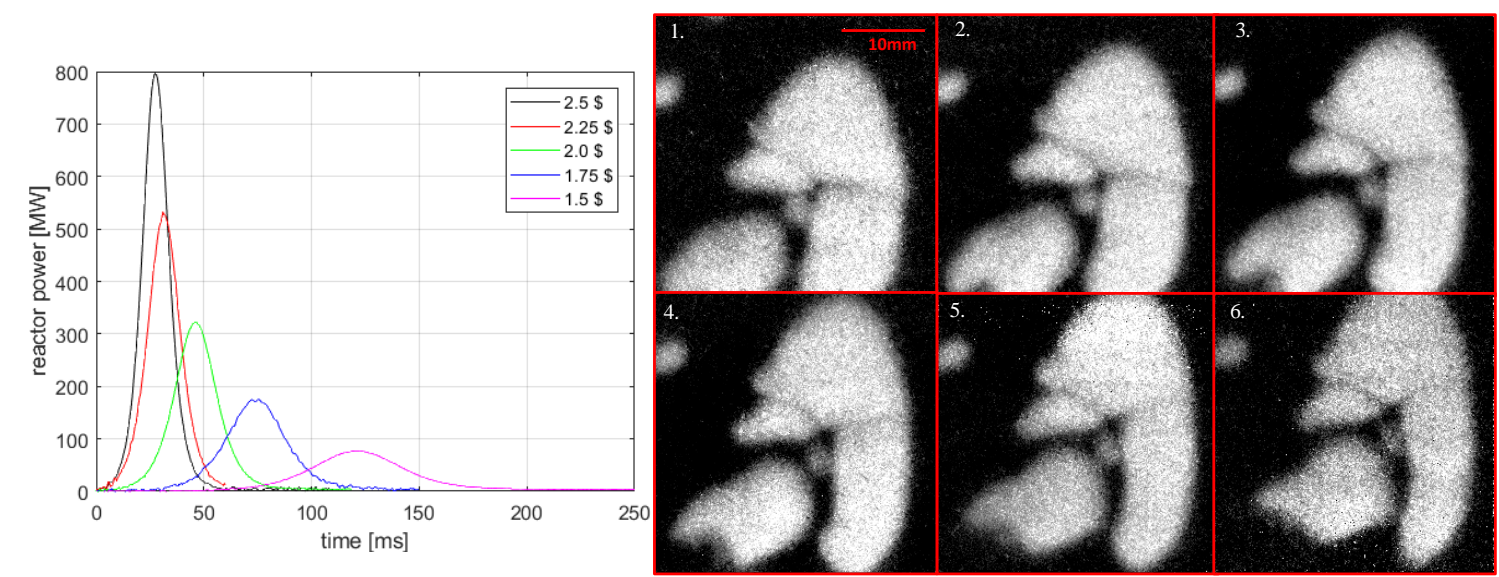

Fig. 5: Left: measured pulse shape at PSBR for a set for different reactivity insertions. Right: image sequence of bubbly, two-phase flow taken at $4 \mathrm{kfps}$ for a $2.5 \$$ pulse. The sequence 1.-6. shows the instantaneous gas volume fraction distribution (white-gas, black-liquid) for every $10^{\text {th }}$ image taken, i.e. with a time gap of $2.5 \mathrm{~ms}$. Image frames are colored red for better visualization.



Fig. 6: Photo (left) and fast neutron radiograph (right) of a Li-filled power source. Imaging as done at the old PSBR beam line using a $3.8 \mathrm{~mm}$ thick PP/ZnS:Cu screen.

As the new beam line leaves the biological shield and enters the new beam cave, we plan to place a rotary beam shutter as is shown on the right of Fig. 2. The rotary beam shutter will have several apertures enabling an easy and fast shutting down of the beam and a flexible in-beam filtering. One aperture will be open for normal beam operation and it will also give access to the removable sapphire filter seated deeper inside the beam port towards the reactor core. This aperture could also house a removable Mirrobor filter for fast neutron imaging. Another aperture will shut the beam down, while a third one will have a few mm thick Cd plate filter to enable epithermal neutron imaging. Switching easily from a thermal to an epithermal beam could have a lot of interesting applications for dual-spectrum or differential imaging of samples that contains component(s) with distinctly different attenuation coefficient e.g. strong resonance(s) in the 
epithermal range. Those components could be quantified by differential imaging. Epithermal imaging is also promising alternative for samples that are slightly too attenuating from thermal neutrons but not that much that only fast neutrons could be used to examine them.

\section{Summary}

The Penn State Breazeale Reactor has just recently undergone a major refurbishment. A new core-moderator assembly has been designed and built enabling a lot of new capabilities or more optimal conditions for neutron beam science. One of the major topics that is being extensively and dynamically developed is neutron imaging. New imaging beam lines are going to be established with thermal and cold spectra. A major effort is also ongoing on imaging detector and imaging methodology development. These include besides general state-of-the-art thermal neutron imaging high-speed, bright flash neutron radiography, fast neutron imaging and dual/multi-spectrum imaging such as thermal/epithermal differential imaging. First results and the corresponding developments have been reported here.

\section{Acknowledgement}

The author is indebted to Mr. Andrew Bascom for providing the results of his MCNP simulations for the flux in new imaging beam port. The author is grateful to Prof. Kenan Unlu, the director of RSEC, for his continued interest and support of his work.

\section{References}

[1] J. Brenizer, M.M. Mench, K. Ünlü, K. Heller, A. Turhan, L. Shi, J.J. Kowal, available at http://www.rsec.psu.edu (accessed July 10th, 2018)

[2] J.M. Cimbala, J.S. Brenizer, A. Po-Ya Chuang, S. Hanna, C. Thomas Conroy, A. El-Ganayni, et al. "Study of a loop heat pipe using neutron radiography” Appl Rad. Iso., 61 (2004), pp. 701705. https://doi.org/10.1016/j.apradiso.2004.03.104

[3] D. Ucar, "Modeling and Design of a new core-moderator assembly and neutron beam ports for the Penn State Breazeale nuclear reactor” Ph.D. Thesis, The Pennsylvania State University, 2013.

[4] http://www.neutronoptics.com/cameras.html

[5] Scintacor, Neutron Screens, available at https://scintacor.com/wpcontent/uploads/2015/09/Datasheet-Neutron-Screens-High-Res.pdf, (accessed on July 20th, 2018)

[6] D. L. Hetrick, Dynamics of Nuclear Reactors, University of Chicago Press, 1971

[7] RC Tritec AG, T., 2017. Scintillators. http://www.rctritec.com/en/scintillators.html.

[8] MirroBor, 2012. Radiation shielding. http://www.mirrotron.kfkipark.hu/shield.html.

[9] D. L. Hetrick, Dynamics of Nuclear Reactors, University of Chicago Press, 1971 